\title{
Manejo do etileno durante o armazenamento de ameixas 'Laetitia’ em atmosfera controlada
}

\author{
Ethylene management during controlled atmosphere storage of 'Laetitia' plums
}

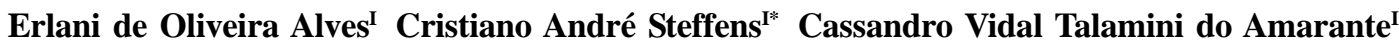 \\ Elizandra Pivotto Pavanello ${ }^{\text {II }}$ Auri Brackmann" ${ }^{\text {II }}$
}

\section{RESUMO}

Objetivou-se avaliar o efeito da aplicação de 1metilciclopropeno (1-MCP) e de diferentes concentrações de etileno sobre a qualidade de ameixas 'Laetitia' armazenadas em atmosfera controlada $(A C)$. Os tratamentos utilizados foram aplicação de 1-MCP (1,0 $\left.\mu L L^{-1}\right)$ e etileno $\left(\mathrm{C}_{2} \mathrm{H}_{4}\right)$ nas concentrações de $<0,04 \mu \mathrm{L} L^{-1}, 1,0 \mu L L^{-1}$ e $10 \mu L L^{-1}$ no interior das câmaras de $\mathrm{AC}\left(1,0 \mathrm{kPa}\right.$ de $\mathrm{O}_{2}+3,0 \mathrm{kPa}$ de $\mathrm{CO}_{2} / 0,5^{\circ} \mathrm{C} \pm 0,1^{\circ} \mathrm{C}$ e UR de $95 \pm 2 \%$ ). A taxa respiratória foi menor nos frutos tratados com 1-MCP e armazenados com $10 \mu L^{-1}$ de $C_{2} H_{1}$. A produção de etileno foi menor nos frutos tratados com 1-MCP. $\mathrm{Na}$ saída da câmara, os frutos tratados com 1-MCP ou armazenados com $<0,04 \mu \mathrm{L} L^{-1}$ de $\mathrm{C}_{2} \mathrm{H}_{4}$ apresentaram maior firmeza de polpa, resistência do fruto à compressão e menor incidência de degenerescência da polpa. Após quatro dias de exposição dos frutos em condição ambiente, a maior firmeza de polpa ocorreu nos frutos tratados com 1-MCP. A acidez

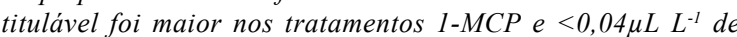
$\mathrm{C}_{2} \mathrm{H}_{4}$. A aplicação de 1-MCP e a absorção de etileno retardam o amadurecimento da ameixa 'Laetitia' e diminuem a incidência de degenerescência da polpa, sendo que o efeito do 1-MCP se prolonga durante o periodo de prateleira. Contudo, mesmo com o uso dessas tecnologias, a incidência de degenerescência de polpa não permite o armazenamento por 60 dias, na condição de AC utilizada.

Palavras-chave: Prunus salicina, amadurecimento, fisiologia pós-colheita, distúrbios fisiológicos.

\section{ABSTRACT}

The objective of this research was to evaluate the effects of treatment with 1-methylcyclopropene (1-MCP) and different ethylene concentrations in the storage room on $f$ 'Laetitia' plums quality stored under controlled atmosphere. The treatments evaluated were 1-MCP application $\left(1.0 \mu \mathrm{L} \mathrm{L}^{-1}\right)$ and ethylene $\left(\mathrm{C}_{2} \mathrm{H}_{4}\right)$ in the concentrations $<0.04 \mu \mathrm{L} \mathrm{L} \mathrm{L}^{-1}, 1.0 \mu \mathrm{L} \mathrm{L} \mathrm{L}^{-1}$, and $10 \mu \mathrm{L} \mathrm{L}^{-1}$ inside the CA storage $\left(1.0 \mathrm{kPa}\right.$ of $\mathrm{O}_{2}+3.0 \mathrm{kPa}$ of $\mathrm{CO}_{2} / 0.5^{\circ} \mathrm{C} \pm 0.1^{\circ} \mathrm{C}$ and $\left.95 \pm 2 \% \mathrm{RH}\right)$ of. Respiratory rates were lower in fruits treated with $1-M C P$ and stored at $10 \mu L L^{-1}$ of $\mathrm{C}_{2} \mathrm{H}_{4}$. Fruits treated with 1-MCP showed the lowest ethylene production rates. At removal from $C A$ storage, fruits treated with 1-MCP and stored at $\mathrm{C}_{2} \mathrm{H}_{4}$ concentration $<0.04 \mu \mathrm{L} \mathrm{L} \mathrm{L}^{-1}$ showed higher flesh firmness and fruit compression resistance and lower flesh browning incidence. After shelf life, fruits treated with 1MCP had the highest flesh firmness. Titratable acidity was higher in fruit treated with 1-MCP and left in CA storage with $<0.04 \mu \mathrm{L}$ $L^{-1} \mathrm{C}_{2} \mathrm{H}_{4}$ concentration. 1-MCP and ethylene absorption delay ripening and reduce flesh browning incidence in 'Laetitia' plums. The effects of 1-MCP persist during shelf life. However, even with the use of these technologies, flesh browning occurrence does not allow fruit storage for 60 days under de CA storage condition used.

Key words: Prunus salicina, ripening, postharvest physiology, physiological disorders.

\section{INTRODUÇÃO}

A atmosfera controlada (AC) é um sistema de armazenamento que permite prolongar o tempo de vida pós-colheita dos frutos, pois ocorre um retardo nos processos relacionados com o amadurecimento, mantendo a qualidade e prolongando o período de prateleira (NAVA \& BRACKMANN, 2001). Em frutos climatéricos, o período máximo de armazenamento depende de processos que são influenciados pelo etileno, como a taxa respiratória, a produção de etileno, o amolecimento e a incidência de distúrbios fisiológicos e de podridões (RATO et al., 2004;

'Departamento de Agronomia, Universidade do Estado de Santa Catarina (UDESC). Av. Luis de Camões, 2090, 88520-000, Bairro Conta Dinheiro, Lages, SC, Brasil. E-mail: steffens@cav.udesc.br. *Autor para correspondência.

IIDepartamento de Fitotecnia, Universidade Federal de Santa Maria (UFSM), Santa Maria, RS, Brasil. 
STEFFENS et al., 2007). No entanto, os efeitos do etileno dependem de sua concentração, a qual induz o amadurecimento somente quando estiver acima de uma concentração considerada fisiologicamente ativa (BRACKMANN et al., 2003). A ameixa 'Laetitia' possui sensibilidade ao etileno (ARGENTA et al., 2003); contudo, não se conhece a concentração a partir da qual o amadurecimento é induzido. O conhecimento dessa concentração crítica é de extrema importância, pois pode servir como ferramenta de orientação para o manejo adequado do etileno durante o armazenamento dessa cultivar.

Alguns pequenos e médios fruticultores utilizam uma mesma câmara de armazenamento para ameixas 'Laetitia' e maçãs 'Gala'. Como esta cultivar de maçã produz grandes quantidades de etileno, a concentração desse regulador vegetal pode atingir valores acima de $20 \mu \mathrm{L} \mathrm{L}^{-1}$ (BRACKMANN et al., 2000) e induzir o rápido amadurecimento das ameixas. Além disso, uma alta sensibilidade do fruto ao etileno em AC indica que há necessidade de utilização de técnicas complementares para minimizar os seus efeitos durante o armazenamento, como o uso de inibidores da ação do etileno (WATKINS, 2006) ou a sua eliminação do ambiente (WILLS et al., 2000).

O 1-metilciclopropeno (1-MCP), um potente inibidor da ação do etileno, constitui-se em uma excelente alternativa para o armazenamento de frutos (WATKINS, 2006). O uso do 1-MCP em ameixas 'Laetitia' mantidas em armazenamento refrigerado apresentou resultado positivo no atraso do amadurecimento (ARGENTA et al., 2003). No entanto, seu efeito em armazenamento em AC é desconhecido. Em maçãs 'Gala', foi observado que o 1-MCP, apesar de ter excelente efeito no armazenamento refrigerado, apresenta menor eficiência em AC, com resultados semelhantes à absorção de etileno (BRACKMANN et al., 2008). Já a eliminação do etileno, no ambiente de armazenamento dos frutos, apresenta excelentes resultados na manutenção da qualidade de maçãs 'Royal Gala' (BRACKMANN et al., 2000) e 'Gala' (BRACKMANN et al., 2008). Em maçãs 'Gala', essa técnica pode apresentar resultados semelhantes à aplicação do 1-MCP na manutenção da qualidade de frutos armazenados em AC, porém com menor custo (BRACKMANN et al., 2008).

O objetivo deste trabalho foi avaliar o efeito de diferentes concentrações de etileno e da aplicação do 1-MCP sobre a qualidade de ameixas 'Laetitia' armazenadas em AC.

\section{MATERIAL E MÉTODOS}

Ameixas 'Laetitia' foram colhidas em pomar comercial localizado no município de Lages, SC, e em seguida transportadas para o Laboratório do Núcleo de Pesquisa em Pós-Colheita - NPP, da Universidade Federal de Santa Maria, localizado no município de Santa Maria, RS. No laboratório, os frutos foram selecionados, eliminando-se aqueles com lesões, defeitos, ferimentos ou danos mecânicos. Posteriormente, foi instalado um experimento em delineamento inteiramente casualizado, com cinco repetições, sendo a unidade experimental constituída por 30 frutos. Os tratamentos utilizados foram: aplicação de 1-MCP $\left(1,0 \mu \mathrm{L} \mathrm{L}^{-1}\right)$ e as concentrações de $\mathrm{C}_{2} \mathrm{H}_{4}$ correspondentes a $<0,04 \mu \mathrm{L} \mathrm{L}^{-1}, 1,0 \mu \mathrm{L} \mathrm{L}^{-1}$ e $10 \mu \mathrm{L} \mathrm{L}^{-1}$. Os frutos foram armazenados em minicâmaras experimentais de AC com capacidade de 180L, procedendo-se à homogeneização das unidades experimentais. A condição de armazenamento utilizada foi de $1,0 \mathrm{kPa}$ de $\mathrm{O}_{2}+3,0 \mathrm{kPa}$ de $\mathrm{CO}_{2}$ na temperatura de $0,5 \pm 0,1^{\circ} \mathrm{C}$ e umidade relativa do ar de $95 \pm 2 \%$.

As pressões parciais dos gases foram obtidas pela diluição do $\mathrm{O}_{2}$ nas minicâmaras com injeção de $\mathrm{N}_{2}$ proveniente de um gerador de nitrogênio que utiliza o princípio Pressure Swing Adsorption (PSA). A manutenção das pressões parciais dos gases nas diferentes câmaras, que variavam em função da respiração dos frutos, foi realizada através da análise diária, com o uso de equipamento automático para controle de gases da marca Kronenberger/Climasul. Quando os níveis do $\mathrm{CO}_{2}$ e $\mathrm{O}_{2}$ não estavam adequados, o equipamento procedia à correção das pressões parciais até os níveis preestabelecidos. $\mathrm{O} \mathrm{O}_{2}$ consumido pela respiração foi reposto por meio da injeção de ar atmosférico nas minicâmaras. $\mathrm{O} \mathrm{CO}_{2}$ em excesso foi absorvido por uma solução de hidróxido de potássio $(40 \%)$, por meio da qual foram circulados os gases das microcâmaras.

Para a eliminação de etileno, no tratamento com baixo etileno $\left(<0,04 \mu \mathrm{L} \mathrm{L}^{-1}\right)$, foi realizada a absorção química desse gás por meio da adição, no interior da microcâmara, de sachês (um sachê para cada $3 \mathrm{~kg}$ de frutos) contendo permanganato de potássio. Para o tratamento com concentração de $1,0 \mu \mathrm{L} \mathrm{L}^{-1}$ de etileno, não foi realizada a absorção. No tratamento com concentração de etileno de $10 \mu \mathrm{L} \mathrm{L}^{-1}$, foi realizada a injeção no interior da microcâmara de gás Etil 5 (5\% de etileno $+95 \%$ de $\mathrm{N}_{2}$ ), proveniente de cilindro de alta pressão, para manter a concentração de etileno preestabelecida, simulando uma situação em que os frutos seriam armazenados juntamente com maçãs 'Gala', o que é muito praticado por pequenos e médios fruticultores.

Ciência Rural, v.39, n.9, dez, 2009. 
Os níveis de etileno foram monitorados semanalmente, por cromatografia gasosa, injetandose $1 \mathrm{~mL}$ de gás da atmosfera de cada câmara em um cromatógrafo a gás, marca Varian, modelo 3400CX, equipado com detector de ionização de chama, coluna empacotada com Porapak $\mathrm{N}$ de $0,7 \mathrm{~m}$ de comprimento, utilizando nitrogênio como gás de arraste. As temperaturas empregadas foram $90^{\circ} \mathrm{C}, 140^{\circ} \mathrm{C}$ e $200^{\circ} \mathrm{C}$ para coluna, injetor e detector, respectivamente.

Para o tratamento com 1-MCP, foi utilizado o produto $\operatorname{SmartFresh}^{\circledR}(0,14 \%$ de 1-MCP na formulação pó), na relação de $0,16 \mathrm{~g}$ de produto $1000 \mathrm{~L}^{-1}$ de câmara, para obter $1,0 \mu \mathrm{LL}^{-1}$ de 1-MCP. O produto foi solubilizado em água, em condição ambiente, em um recipiente hermético, e, posteriormente, a solução foi transferida para uma placa de Petri dentro de uma minicâmara com volume de $180 \mathrm{~L}$, com fechamento hermético. Os frutos ficaram expostos ao tratamento por 24 horas.

Após 60 dias de armazenamento, os frutos foram transportados para o Laboratório de Pesquisa em Fisiologia e Tecnologia Pós-Colheita da Universidade do Estado de Santa Catarina (UDESC), e as amostras divididas em duas subamostras de 15 frutos, uma para análise na saída da câmara e outra para análise após quatro dias de exposição dos frutos em condição ambiente $\left(20 \pm 2^{\circ} \mathrm{C} / 60 \pm 5 \%\right.$ de UR).

Foram quantificadas as taxas respiratórias e de produção de etileno, colocando-se 15 frutos de cada amostra em um recipiente com o volume de 2,3L, que permite fechamento hermético. Alíquotas de gás $(1000 \mu \mathrm{L})$ foram retiradas dos recipientes através de um septo e injetadas em um cromatógrafo a gás Varian ${ }^{\circledR}$, modelo CP-3800, equipado com uma coluna Porapak $\mathrm{N}^{\circledR}$ de 3,0m de comprimento (80-100 mesh), metanador e detector de ionização de chama. As temperaturas da coluna, do detector, do metanador e do injetor foram de $45^{\circ} \mathrm{C}, 120^{\circ} \mathrm{C}, 300^{\circ} \mathrm{C}$ e $110^{\circ} \mathrm{C}$, respectivamente. Os fluxos de nitrogênio, hidrogênio e ar sintético foram de 70,30 e $300 \mathrm{~mL} \mathrm{~min}^{-1}$, respectivamente. Os valores das taxas respiratórias e de produção de etileno foram expressos em nmol de $\mathrm{CO}_{2} \mathrm{~kg}^{-1} \mathrm{~s}^{-1}$ e pmol de $\mathrm{C}_{2} \mathrm{H}_{4} \mathrm{~kg}^{-1} \mathrm{~s}^{-1}$, respectivamente. A firmeza de polpa $(\mathrm{N})$ foi determinada na região equatorial dos frutos, em dois lados opostos, após remoção de uma pequena porção da epiderme e com auxílio de um penetrômetro equipado com ponteira de 7,9mm de diâmetro. A acidez titulável (AT; mEq $\left.100 \mathrm{~mL}^{-1}\right)$ foi obtida através de uma amostra de $10 \mathrm{~mL}$ de suco previamente extraído de fatias transversais retiradas da região equatorial dos frutos e trituradas em uma centrífuga. Essa amostra foi diluída em $90 \mathrm{~mL}$ de água destilada e titulada com solução de $\mathrm{NaOH} 0,1 \mathrm{~N}$ até $\mathrm{pH} 8,1$. O teor de sólidos solúveis ( $\mathrm{SS} ;{ }^{\circ} \mathrm{Brix}$ ) foi determinado por refratometria, utilizando-se o suco extraído conforme descrito para a AT, sendo realizada a correção do efeito da temperatura $\left(20^{\circ} \mathrm{C}\right)$. Os atributos de textura foram analisados com um texturômetro eletrônico TAXT-plus ${ }^{\circledR}$ (Stable Micro Systems Ltd., Reino Unido), em termos de força para ruptura da epiderme, para a penetração na polpa e para a compressão do fruto inteiro. Para a quantificação da força para ruptura da epiderme e para a penetração na polpa, foi utilizada uma ponteira, modelo PS2, com mm de diâmetro, sem remoção da epiderme, a qual foi introduzida na polpa a uma profundidade de $5 \mathrm{~mm}$ com velocidades pré-teste, teste e pós-teste de 30,5 e $30 \mathrm{~mm}$ $\mathrm{s}^{-1}$, respectivamente. A resistência do fruto à compressão foi determinada usando-se uma plataforma plana, modelo $\mathrm{P} / 75$, com $75 \mathrm{~mm}$ de diâmetro, que exerceu uma força de compressão até uma deformação de $5 \mathrm{~mm}$ na superfície do fruto. $\mathrm{O}$ índice de cor vermelha (ICV) foi determinado avaliando-se a superfície dos frutos recoberta com coloração vermelha, sendo atribuídas notas de 1 a $4(1,2,3$ e 4 para o fruto com, respectivamente, $0-25 \%, 26-50 \%, 51-75 \%$ e $76-100 \%$ da superfície pigmentada de vermelho). O índice foi calculado pelo somatório dos produtos do número de frutos pela sua respectiva nota, dividido pelo total de frutos da amostra. A cor da epiderme (ângulo hue) foi determinada com um colorímetro Minolta, modelo CR400, sendo as leituras realizadas nos lados mais vermelho e menos vermelho do fruto, na região equatorial. Os resultados foram expressos em ângulo de cor $\left(\mathrm{h}^{\circ}\right) . \mathrm{O} \mathrm{h}^{\circ}$ define a coloração básica, sendo que $0^{\circ}=$ vermelho, $90^{\circ}=$ amarelo e $180^{\circ}=$ verde. A incidência de podridões foi avaliada pela contagem dos frutos afetados, que apresentaram lesões maiores do que $5 \mathrm{~mm}$ de diâmetro, com características de ataque de patógenos. A incidência de degenerescência da polpa foi avaliada através de corte transversal na região equatorial do fruto, sendo contabilizados os frutos que apresentaram escurecimento da polpa, e os resultados expressos em porcentagem (\%).

Os dados foram submetidos à ANOVA com teste F. Dados em porcentagem foram transformados pela fórmula $\operatorname{arcsen}[(\mathrm{x}+0,5) / 100]^{1 / 2}$ antes de serem submetidos à ANOVA. Para a comparação das médias, adotou-se o teste de Tukey a 5\% de probabilidade de erro.

\section{RESULTADOS E DISCUSSÃO}

Os frutos tratados com 1-MCP e armazenados com $10 \mu \mathrm{L} \mathrm{L}^{-1}$ de $\mathrm{C}_{2} \mathrm{H}_{4}$ apresentaram menores taxas respiratória e de produção de etileno (Tabela 1). ARGENTA et al. (2003) também observaram menores taxas respiratória e de produção de etileno em 
Tabela 1 - Taxas respiratórias e de produção de etileno em ameixas 'Laetitia' armazenadas por $60 \mathrm{dias}$ em $\mathrm{AC}\left(1,0 \mathrm{kPa}\right.$ de $\mathrm{O}_{2}+3,0 \mathrm{kPa}$ de $\mathrm{CO}_{2} / 0,5^{\circ} \mathrm{C} \pm 0,1^{\circ} \mathrm{C}$ e UR de $95 \pm 2 \%$ ), com aplicação de $1-\mathrm{MCP}$ e em diferentes concentrações de $\mathrm{C}_{2} \mathrm{H}_{4}$, na saída da câmara frigorífica e após quatro dias em condição ambiente $\left(20 \pm 2^{\circ} \mathrm{C} / 60 \pm 5 \%\right.$ de UR). Lages, 2008.

\begin{tabular}{|c|c|c|c|c|}
\hline \multirow{2}{*}{ Tratamentos } & \multicolumn{2}{|c|}{----Taxa respiratória $\left(\eta \mathrm{mol} \mathrm{CO} \mathrm{Cg}_{2} \mathrm{~kg}^{-1} \mathrm{~s}^{-1}\right)$--- } & \multicolumn{2}{|c|}{---Produção de etileno $\left(\rho \mathrm{mol} \mathrm{C}_{2} \mathrm{H}_{4} \mathrm{~kg}^{-1} \mathrm{~s}^{-1}\right)$------ } \\
\hline & Saída da câmara & $\begin{array}{c}\text { Quatro dias em condição } \\
\text { ambiente }\end{array}$ & Saída da câmara & $\begin{array}{c}\text { Quatro dias em condição } \\
\text { ambiente }\end{array}$ \\
\hline $1-\mathrm{MCP}$ & $192,5 \mathrm{c}$ & $117,7 \mathrm{~b}$ & $0,57 \mathrm{c}$ & $0,33 \mathrm{c}$ \\
\hline$<0,04 \mu \mathrm{L} \mathrm{L}^{-1}$ de $\mathrm{C}_{2} \mathrm{H}_{4}$ & $358,1 \mathrm{~b}$ & $293,0 \mathrm{a}$ & $2,44 b$ & $4,29 \mathrm{a}$ \\
\hline $1 \mu \mathrm{L} \mathrm{L}^{-1}$ de $\mathrm{C}_{2} \mathrm{H}_{4}$ & $727,3 a$ & $298,9 \mathrm{a}$ & $4,39 \mathrm{a}$ & $5,13 \mathrm{a}$ \\
\hline $10 \mu \mathrm{L} \mathrm{L}^{-1}$ de $\mathrm{C}_{2} \mathrm{H}_{4}$ & $253,8 \mathrm{bc}$ & $167,0 \mathrm{~b}$ & $1,28 \mathrm{c}$ & $2,26 \mathrm{~b}$ \\
\hline CV $(\%)$ & 12,8 & 19,0 & 13,5 & 19,9 \\
\hline
\end{tabular}

Médias não seguidas pela mesma letra nas colunas diferem entre si pelo teste de Tukey a 5\% de probabilidade de erro.

ameixas 'Laetitia' tratadas com 1-MCP. O efeito do 1MCP sobre a redução nas taxas respiratória e de produção de etileno está relacionado à inibição da ação do etileno. Em maçãs tratadas com 1-MCP, a menor produção de etileno foi relacionada à menor produção de transcritos das enzimas 1-aminociclopropano carboxilase sintase (ACC sintase) e 1aminociclopropano carboxilase oxidase (ACC oxidase) e dos receptores de etileno, ETR1 e ERS1 (DAL CIN et al., 2006; WATKINS, 2006). Menores taxas respiratórias e de produção de etileno em condições de AC com concentrações maiores que $10 \mu \mathrm{L} \mathrm{L}^{-1}$ de $\mathrm{C}_{2} \mathrm{H}_{4}$ também foram observadas em ameixas 'Red Rose' (DONG et al., 2001). Possivelmente, esse resultado esteja relacionado ao fato de os frutos estarem em estádio mais adiantado de amadurecimento e/ou senescência, pelo efeito do etileno durante o armazenamento. Em frutos climatéricos, esse estádio de desenvolvimento é caracterizado pela redução na atividade respiratória e na biossíntese de etileno. Em maçãs 'Royal Gala', altas concentrações de etileno $\left(>20 \mu \mathrm{L} \mathrm{L}^{-1}\right)$ induziram a senescência mais rapidamente (BRACKMANN et al., 2000).

Os maiores valores de taxas respiratórias e de produção de etileno, na saída da câmara, foram observados em frutos armazenados com $1,0 \mu \mathrm{L} \mathrm{L}^{-1} \mathrm{de}$ $\mathrm{C}_{2} \mathrm{H}_{4}$, sendo que frutos tratados com $<0,04 \mu \mathrm{L} \mathrm{L}^{-1} \mathrm{de}$ $\mathrm{C}_{2} \mathrm{H}_{4}$ apresentaram comportamento intermediário e frutos tratados com $10 \mu \mathrm{L} \mathrm{L}^{-1}$ de $\mathrm{C}_{2} \mathrm{H}_{4}$, os menores valores (Tabela 1). Esses resultados demonstram que o fitormônio acelerou o amadurecimento dos frutos. Em frutos climatérios, ocorre aumento nas taxas respiratória e de produção de etileno durante o amadurecimento, ocorrendo uma queda na fase de senescência (WILLS et al., 2000). Baseando-se nesses resultados, supõe-se que os frutos dos tratamentos com $<0,04 \mu \mathrm{L} \mathrm{L}^{-1}, 1,0 \mu \mathrm{L} \mathrm{L}^{-1}$ e $10 \mu \mathrm{L} \mathrm{L}^{-1}$ de $\mathrm{C}_{2} \mathrm{H}_{4}$ aparentemente encontravam-se nos estádios préclimatérico, climatérico e pós-climatérico, respectivamente.

Na saída da câmara, a firmeza de polpa e a resistência do fruto à compressão apresentaram maiores valores nos frutos armazenados na menor concentração de etileno e naqueles tratados com 1-MCP (Tabela 2). Contudo, as forças para ruptura da epiderme e para penetração da polpa não apresentaram diferenças entre tratamentos. Após quatro dias em condição ambiente, a firmeza de polpa e a força para ruptura da casca foram mais elevadas nos frutos tratados com 1-MCP, sendo que, para os demais atributos de textura, não houve diferenças significativas entre tratamentos (Tabela 2). Esses mesmos efeitos sobre a consistência da polpa foram obtidos com a aplicação de 1-MCP em maçã 'Gala' (BRACKMANN et al., 2004) e ameixa 'Laetitia' (ARGENTA et al., 2003) e com a absorção do etileno em maçã 'Gala' (BRACKMANN et al., 2003) e 'Royal Gala' (BRACKMANN et al., 2000). Possivelmente, esses resultados estão relacionados à menor ação do etileno sobre as enzimas hidrolíticas de parede celular, pois o mesmo é necessário para a atividade de enzimas responsáveis pela redução da consistência dos frutos (JOHNSTON et al., 2001; MAJUMDER \& MAZUMDAR, 2002).

Apesar das diferenças entre tratamentos para a firmeza de polpa, a variável força para a penetração da polpa não apresentou diferenças significativas entre os tratamentos na saída da câmara e após quatro dias em temperatura ambiente (Tabela 2). Segundo GUILLERMIN et al. (2006), pode ocorrer comportamento diferenciado entre os atributos firmeza de polpa e força para a penetração da polpa. Isso resulta do fato de que a determinação da firmeza de polpa desconsidera a resistência exercida pelo tecido epidérmico e pela camada de células hipodérmicas, tecidos considerados na avaliação da força para penetração da polpa. 
Tabela 2 - Qualidade físico-química de ameixas 'Laetitia' armazenadas por 60 dias em $\mathrm{AC}\left(1,0 \mathrm{kPa}\right.$ de $\mathrm{O}_{2}+3,0 \mathrm{kPa}$ de $\mathrm{CO}_{2} / 0,5^{\circ} \mathrm{C} \pm 0,1^{\circ} \mathrm{C}$ e UR de $95 \pm 2 \%$ ), com aplicação de 1-MCP e em diferentes concentrações de $\mathrm{C}_{2} \mathrm{H}_{4}$, na saída da câmara frigorífica e após quatro dias em condição ambiente $\left(20 \pm 2^{\circ} \mathrm{C} / 60 \pm 5 \%\right.$ de UR). Lages, 2008.

\begin{tabular}{|c|c|c|c|c|c|}
\hline Tratamentos & $\begin{array}{l}\text { Firmeza de polpa } \\
\qquad(\mathrm{N})\end{array}$ & $\begin{array}{l}\text { Acidez titulável } \\
\left(\mathrm{meq} 100 \mathrm{~mL}^{-1}\right)\end{array}$ & $\begin{array}{l}\text { Força para ruptura } \\
\text { da casca }(\mathrm{N})\end{array}$ & $\begin{array}{c}\text { Força para } \\
\text { penetração da polpa } \\
\text { (N) }\end{array}$ & $\begin{array}{l}\text { Resistência do fruto } \\
\text { à compressão }(\mathrm{N})\end{array}$ \\
\hline & \multicolumn{5}{|c|}{ Saída da câmara } \\
\hline $1-\mathrm{MCP}$ & $37,1 \mathrm{ab}$ & $17,9^{\mathrm{a}}$ & $10,6 \mathrm{a}$ & $2,33 \mathrm{a}$ & $95,0 \mathrm{ab}$ \\
\hline$<0,04 \mu \mathrm{L} \mathrm{L}^{-1} \mathrm{de} \mathrm{C}_{2} \mathrm{H}_{4}$ & $38,4 \mathrm{a}$ & $19,0 \mathrm{a}$ & $10,2 \mathrm{a}$ & $2,10 \mathrm{a}$ & $105,7 \mathrm{a}$ \\
\hline $1 \mu \mathrm{L} \mathrm{L}^{-1}$ de $\mathrm{C}_{2} \mathrm{H}_{4}$ & $35,6 a b$ & $19,4^{\mathrm{a}}$ & $9,6 \mathrm{a}$ & $2,56 \mathrm{a}$ & $78,3 \mathrm{~b}$ \\
\hline $10 \mu \mathrm{L} \mathrm{L}^{-1}$ de $\mathrm{C}_{2} \mathrm{H}_{4}$ & $32,5 b$ & $17,8^{\mathrm{a}}$ & $9,7 \mathrm{a}$ & $1,86 \mathrm{a}$ & $77,0 \mathrm{~b}$ \\
\hline \multirow[t]{2}{*}{$\mathrm{CV}(\%)$} & 6,6 & 4,9 & 7,4 & 10,3 & 12,0 \\
\hline & \multicolumn{5}{|c|}{ Quatro dias em condição ambiente } \\
\hline $1-\mathrm{MCP}$ & $39,7 \mathrm{a}$ & $20,5 \mathrm{a}$ & $11,1 \mathrm{a}$ & $2,26 \mathrm{a}$ & $83,1 \mathrm{a}$ \\
\hline$<0,04 \mu \mathrm{L} \mathrm{L}^{-1}$ de $\mathrm{C}_{2} \mathrm{H}_{4}$ & $32,7 b$ & $21,4 \mathrm{a}$ & $10,4 b$ & $2,24 \mathrm{a}$ & $86,7 \mathrm{a}$ \\
\hline $1 \mu \mathrm{L} \mathrm{L}^{-1}$ de $\mathrm{C}_{2} \mathrm{H}_{4}$ & $33,8 b$ & $15,6 b$ & $10,1 b$ & $2,04 \mathrm{a}$ & $74,4 \mathrm{a}$ \\
\hline $10 \mu \mathrm{L} \mathrm{L}^{-1}$ de $\mathrm{C}_{2} \mathrm{H}_{4}$ & $30,7 b$ & $17,8 \mathrm{~b}$ & $10,0 \mathrm{~b}$ & $2,15 \mathrm{a}$ & $79,9 \mathrm{a}$ \\
\hline $\mathrm{CV}(\%)$ & 8,7 & 5,2 & 4,2 & 8,7 & 9,2 \\
\hline
\end{tabular}

Médias não seguidas pela mesma letra nas colunas diferem entre si pelo teste de Tukey a 5\% de probabilidade de erro.

Após 60 dias de armazenamento da ameixa 'Laetitia', verificou-se que, na saída da câmara, não houve diferença entre tratamentos para AT. Contudo, após quatro dias em condição ambiente, os maiores valores de AT foram observados nos frutos tratados com 1-MCP e armazenados na concentração de etileno $<0,04 \mu \mathrm{L} \mathrm{L}^{-1}$ (Tabela 2), demonstrando um atraso no amadurecimento dos frutos. ARGENTA et al. (2003) também observaram maior AT em ameixas 'Laetitia' tratadas com 1-MCP. O efeito da eliminação do etileno sobre a manutenção da AT também foi observado em maçãs 'Royal Gala' (BRACKMANN et al., 2000) e 'Gala' (BRACKMANN et al., 2003).

A cor, avaliada em termos de índice de cor vermelha e $\mathrm{h}^{\circ}$, nos lados mais e menos vermelhos dos frutos, não foi influenciada pelos tratamentos (Tabela 3). No entanto, alguns trabalhos demonstram que alterações na coloração em ameixas, durante o amadurecimento, são processos dependentes de etileno (ARGENTA et al., 2003; PALOU \& CRISOSTO, 2003).

Tabela 3 - Cor e degenerescência da polpa em ameixas 'Laetitia' armazenadas por 60 dias em $\mathrm{AC}\left(1,0 \mathrm{kPa}\right.$ de $\mathrm{O}_{2}+3,0 \mathrm{kPa}$ de $\mathrm{CO}_{2} /$ $0,5^{\circ} \mathrm{C} \pm 0,1^{\circ} \mathrm{C}$ e UR de $9 \pm 2 \%$ ), com aplicação de 1-MCP e em diferentes concentrações de $\mathrm{C}_{2} \mathrm{H}_{4}$, na saída da câmara frigorífica e após quatro dias em condição ambiente $\left(20 \pm 2^{\circ} \mathrm{C} / 60 \pm 5 \%\right.$ de UR). Lages, 2008.

\begin{tabular}{|c|c|c|c|c|}
\hline \multirow{3}{*}{ Tratamentos } & \multirow{3}{*}{ Índice de cor vermelha* (1-4) } & \multicolumn{2}{|c|}{ Ângulo hue $\left(\mathrm{h}^{\circ}\right)$} & \multirow{3}{*}{ Degenerescência da polpa $(\%)$} \\
\hline & & & & \\
\hline & & Lado mais vermelho & Lado menos vermelho & \\
\hline & \multicolumn{4}{|c|}{-----------------------------------------------------------Saída da câmara---------------------------------------------------------- } \\
\hline 1-MCP & $2,64 \mathrm{a}$ & $37,5 \mathrm{a}$ & $64,8 \mathrm{a}$ & $16,6 \mathrm{~b}$ \\
\hline$<0,04 \mu \mathrm{L} \mathrm{L}^{-1}$ de $\mathrm{C}_{2} \mathrm{H}_{4}$ & $2,70 \mathrm{a}$ & $41,2 \mathrm{a}$ & $82,7 \mathrm{a}$ & $18,5 \mathrm{~b}$ \\
\hline $1 \mu \mathrm{L} \mathrm{L}^{-1} \mathrm{de} \mathrm{C}_{2} \mathrm{H}_{4}$ & $2,81 \mathrm{a}$ & $35,6 \mathrm{a}$ & $91,2 \mathrm{a}$ & $53,9 \mathrm{a}$ \\
\hline $10 \mu \mathrm{L} \mathrm{L}^{-1}$ de $\mathrm{C}_{2} \mathrm{H}_{4}$ & $2,95 \mathrm{a}$ & $39,8 \mathrm{a}$ & $83,9 \mathrm{a}$ & $39,9 a$ \\
\hline \multirow[t]{2}{*}{$\mathrm{CV}(\%)$} & 6,6 & 9,4 & 23,4 & 23,3 \\
\hline & & -----Quatro dias em & ondição ambiente-------- & 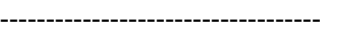 \\
\hline $1-\mathrm{MCP}$ & $3,78 \mathrm{a}$ & $30,8 \mathrm{a}$ & $59,7 \mathrm{a}$ & $41,3 \mathrm{a}$ \\
\hline$<0,04 \mu \mathrm{L} \mathrm{L}^{-1} \mathrm{de}_{2} \mathrm{H}_{4}$ & $3,65 \mathrm{a}$ & $29,8 \mathrm{a}$ & $64,8 \mathrm{a}$ & $37,3 \mathrm{a}$ \\
\hline $1 \mu \mathrm{L} \mathrm{L}^{-1}$ de $\mathrm{C}_{2} \mathrm{H}_{4}$ & $3,85 \mathrm{a}$ & $30,6 \mathrm{a}$ & $61,5 \mathrm{a}$ & $48,9 \mathrm{a}$ \\
\hline $10 \mu \mathrm{L} \mathrm{L}^{-1}$ de $\mathrm{C}_{2} \mathrm{H}_{4}$ & $3,70 \mathrm{a}$ & $31,6 \mathrm{a}$ & $61,0 \mathrm{a}$ & $27,5 \mathrm{a}$ \\
\hline $\mathrm{CV}(\%)$ & 19,0 & 5,2 & 13,5 & 37,7 \\
\hline
\end{tabular}

Médias não seguidas pela mesma letra nas colunas diferem entre si pelo teste de Tukey a 5\% de probabilidade de erro.*1=0-25\%; $2=26-50 \%$; $3=51-75 \%$; e $4=>75 \%$ da superfície recoberta com cor vermelha.

Ciência Rural, v.39, n.9, dez, 2009. 
A maior incidência de degenerescência da polpa foi observada nos tratamentos com $1,0 \mu \mathrm{L} \mathrm{L}^{-1} \mathrm{e}$ $10,0 \mu \mathrm{L} \mathrm{L}^{-1}$ de etileno (Tabela 3). Esse resultado demonstra que a degenerescência da polpa, além da baixa temperatura e do tempo de armazenamento, está relacionada à ação do etileno. ARGENTA et al. (2003) constataram redução na incidência de degenerescência da polpa em ameixas 'Laetitia' com o uso do 1-MCP. BRACKMANN et al. (2000) observaram menor incidência do distúrbio em maçãs 'Royal Gala' armazenadas em menores concentrações de etileno. Contudo, no presente estudo, apesar da redução na incidência de degenerescência da polpa com o uso do 1-MCP e com a absorção de etileno, observou-se incidência desse distúrbio em todos os tratamentos. Isso pode estar relacionado ao tempo de armazenamento prolongado ou à condição de $\mathrm{AC}$ inadequada para o armazenamento dessa cultivar.

A incidência de podridões e os teores de SS, tanto na saída da câmara, como após quatro dias de exposição dos frutos em condição ambiente, não apresentaram diferenças entre tratamentos (dados não apresentados).

\section{CONCLUSÃO}

A aplicação de 1-MCP e a absorção de etileno retardam o amadurecimento da ameixa 'Laetitia' e diminuem a incidência de degenerescência da polpa, sendo que o efeito do 1-MCP se prolonga durante o período de prateleira. Contudo, mesmo com o uso dessas tecnologias, a alta incidência de degenerescência de polpa não permite o armazenamento por 60 dias na condição de $1,0 \mathrm{kPa}$ de $\mathrm{O}_{2}$ e $3,0 \mathrm{kPa}$ de $\mathrm{CO}_{2}$ a $0,5^{\circ} \mathrm{C}$.

\section{AGRADECIMENTOS}

Os autores agradecem ao Conselho Nacional de Desenvolvimento Científico e Tecnológico $(\mathrm{CNPq})$, à Fundação de Amparo à Pesquisa e Desenvolvimento Científico e Tecnológico de Santa Catarina (FAPESC) e à Coordenação de Aperfeiçoamento de Pessoal de Nível Superior (CAPES), pelo apoio financeiro a este projeto.

\section{REFERÊNCIAS}

ARGENTA, L.C. et al. Ripening and quality of 'Laetitia' plums following harvest and cold storage as affected by inhibition of ethylene action. Pesquisa Agropecuária Brasileira, Brasília, v.38, n.10, p.1139-1148, 2003. Disponível em: <http:// w w w. s c i e 1 o.b r / s c i e 1 o.p h p ? p i d = S $0100-$ $204 X 2003001000002 \&$ script $=$ sci_arttext $>$. Acesso em $04 \mathrm{de}$ ago de 2009. doi: 10.1590/S010̄0-204X2003001000002.

BRACKMANN, A. et al. Armazenamento de maçãs 'Royal Gala' sob diferentes concentrações de etileno. Revista Brasileira de Agrociência, Pelotas, v.6, n.1, p.39-41, 2000.
BRACKMANN, A. et al. Armazenamento de maçã 'Gala' em atmosfera controlada com remoção do etileno. Ciência Rural, Santa Maria, v.33, n.4, p.647-650, 2003. Disponível em: $<$ http://www.scielo.br/scielo.php?pid=S 0103 $84782005000200037 \&$ script $=$ sci_arttext $>$. Acesso em $04 \mathrm{de}$ ago de 2009. doi: 10.1590/S0103-84782005000200037.

BRACKMANN, A. et al. Qualidade da maçã cv. Gala tratada com 1metilciclopropeno. Ciência Rural, Santa Maria, v.34, n.5, p.1415-1420, 2004. Disponível em: <http://www.scielo.br/scielo.php?pid=S0103$84782004000500014 \&$ script $=$ sci_abstract\&tlng-pt $>$. Acesso em: 23 de jun 2009. doi: 10.1590/S0103-84782004000500014.

BRACKMANN, A. et al. Qualidade da maçã 'Gala' armazenada em atmosfera controlada associada a absorção e ao controle da síntese e da ação do etileno. Ciência Rural, Santa Maria, v.38, n.8, p.2151-2156, 2008. Disponível em: <http:// w w w. s c i e 1 o.b r / s c i e 1 o.p h p ? p i d = S $0100-$ $204 X 2003001000002 \&$ script $=$ sci_arttext $>$. Acesso em $04 \mathrm{de}$ ago de 2009. doi: 10.1590/S0100-204X2003001000002.

DAL CIN, V. et al. The ethylene biosynthetic and signal transduction pathways are differently affected by 1-MCP in apple and peach fruit. Postharvest Biology and Technology, Amsterdam, v.42, n.2, p.125133, 2006. Disponível em: <http:/www.sciencedirect.com/ science?_ob=ArticleURL\&_udi=B6TBJ-4KYY3R6$2 \&$ _user $=686176 \&$ _rdoc $=1 \&$ fmt $=\&$ \&orig $=$ search $\&$ _sort $=\mathrm{d} \&$ d ocanchor $=\&$ view $=c \&$ c searchStrId $=937719886 \&$ rerunOrigin $=$ google\&_acct $=$ C000037169\&_version $=1 \&$ \&urlVersion $=0$ \&_userid $=686176 \& \mathrm{md} 5=\mathrm{f5e} 3578 \mathrm{da} 2313 \mathrm{cf5ce} 5346 \mathrm{f5} 560 \mathrm{f} 94 \mathrm{be}>$. Acesso em: 23 de jun 2009. doi:10.1016/j.postharvbio.2006.06.008.

DONG, L. et al. Ripening of 'Red Rosa' plums: effect of ethylene and 1-metilciclopropene. Journal of Plant Physiology, Collingwood, v.28, n.7, p.1039-1045, 2001.

GUILLERMIN, P. et al. Rheological and technological properties of two cider apple cultivars. LWT - Food Science and Technology, Amsterdam, v.39, n.9, p.995-1000, 2006. Disponível em: \http://www.sciencedirect.com/ science?_ob=ArticleURL\&_udi=B6WMV-4JRVCRD$2 \&$ _user $=686176 \&$ rdoc $=1 \&$ fmt $=\&$ \&orig $=$ search\&_sort $=\mathrm{d} \&$ d ocanchor $=\&$ view $=$ c \&_searchStrId $=973948102 \&$ _rerunOrigin $=$ google\&_acct $=$ C000037169\&_version $=1 \&$ _urlVersion $=$ $0 \&$ userid $=686176 \& \mathrm{md} 5=$ fbb0f5343940dded $7068196 \mathrm{~b} 9 \mathrm{ec} 67 \mathrm{c} 9 \mathrm{~b}>$. Acesso em: 23 de jun 2009. doi: 10.1016/j.lwt.2006.02.025.

JOHNSTON, J.W. et al. Temperature induces differential softening responses in apple cultivars. Postharvest Biology and Technology, Amesterdam, v.23, n.3, p.185-196, 2001.

MAJUMDER, K.; MAZUMDAR, B.C. Changes of pectic substances in developing fruits of cape-gooseberry (Physalis peruviana L.) in relation to the enzyme activity and evolution of ethylene. Scientia Horticulturae, Amsterdam, v.96, n.14, p.91-101, 2002.

NAVA, A.G.; BRACKMANN, A. Efeito da remoção do etileno e sistema de armazenamento sobre a qualidade de pêssegos [Prunus persica (L). Batsch], cv. 'Chiripá'. Revista Brasileira de Agrociência, Pelotas, v.7, n.2, p.153-158, 2001. 
PALOU, L.; CRISOSTO, C.H. The influence of exogenous ethylene application during cold storage on stone fruit quality and brown rot development. Acta Horticulturae, Leuven, n.628, p.269-276, 2003 .

RATO, A.R. et al. Produção de etileno em frutos de ameixeira Prunus domestica sujeita a duas condições de temperatura. Revista Ciências Agrárias, Lisboa, v.30, n.1, p.331-338, 2004.

STEFFENS, C.A. et al. Taxa respiratória de frutas de clima temperado. Pesquisa Agropecuária Brasileira, Brasília, v.42, n.3, p.313-321, 2007. Disponível em: <http:// www.scielo.br/pdf/pab/v42n3/03.pdf $>$. Acesso em 04 de ago de 2009. doi: 10.1590/S0100-204X2007000300003.
WATKINS, C.B. The use of 1-methylcyclopropene (1-MCP) on fruits and vegetables. Biotechnology Advances, Amsterdam, n.24, p.389-409, 2006. Disponível em: <http://www.sciencedirect.com/ science?_ob=ArticleURL\&_udi=B6T4X-4JFGFB 4 $1 \&$ _user $=686176 \&$ rdoc $=1 \&$ fmt $=\&$ \&orig $=$ search $\&$ sort $=\mathrm{d} \&$ _docanchor $=\&$ view $=c \&$ csearchStrId $=937721975 \&$ _rerun Origin=google\&_acct $=\mathrm{C} 000037169 \&$ _version $=1$ \&_urlVersion $=0 \&$ userid $=-686176 \& \mathrm{md} 5=4565996 \mathrm{e} 51 \mathrm{cc} 8668966 \mathrm{dc} 85 \mathrm{fe} 2 \mathrm{ef} 07 \mathrm{~b}>$. Acesso em: 23 de jun 2009. doi:10.1016/j.biotechadv.2006.01.005.

WILLS, R.B. et al. Ethylene levels associated with fruit and vegetables during marketing. Australian Journal of Experimental Agriculture, Collingwood, v.40, p.467-470, 2000 . 